\title{
Hydrochlorothiazide Nanocrystals Stabilization by Silk Sericin
}

\author{
Priyanka Giri, Poonam Jadhav, Udaykumar Patil, Namdeo R. Jadhav*
}

Department of Pharmaceutics, Bharati Vidyapeeth College of Pharmacy, Kolhapur, Maharashtra, INDIA.

\begin{abstract}
Aim: The aim of present study deals with preparation and stabilization of Hydrochlorothiazide nanocrystals using silk sericin. Materials and Methods: Nanocrystals of BCS class II drugs were primed by antisolvent precipitation method in ratio of HCTZ: Sericin $(1: 0.5,1: 1,1: 1.5,1: 2)$. Further Nanocrystals were investigated by particle size, zeta potential, drug content, ATR- FTIR, XRD, DSC, SEM, in vitro dissolution study and stability study. Zeta potential $(1: 0.5)$ shows higher value -18.8 than other batches which means optimized P1 batch have more stability than other batches. Low loss of drug content in lyophilized $\mathrm{P} 1$ batch resulted in excellent revival of nanocrystals. In FTIR, principle peaks of pure drug HCTZ were retained in 1 :0.5) P1 batch which are almost identical. Further XRD proved that HCTZ existed in crystalline state in the HCTZ nanocrystals. DSC of HCTZ and HCTZ nanocrystals peaks were found to be nearly indistinguishable. Nanocrystals were found to be sharp needle in shape. Results: Nanocrystals of P1 batch displayed enhanced dissolution performance than other batches as its \% drug dissolution was found to be 89.39 . \% crystallinity of nanocrystals was somewhat increased but not any noteworthy difference after 3 months storage as \% crystainity of P1 batch after storage for 3 months was found to be 44.01 to 45.07 hence it was increased to some extent, but difference was not noteworthy. Conclusion: Result verified that, sericin improves solubility and stability of nanocrystals. Thus, sericin play important role in stabilization of nanocrystals of poorly soluble drugs.
\end{abstract}

Key words: Sericin, Stabilization, Lyophilization, Solubility, Antisolvent, Nanocrystals.

\section{INTRODUCTION}

It is observed that $40 \%$ of examined drugs and about $90 \%$ of approved drugs have shown poor aqueous solubility which resulted in their limited bioavailability.

One of the important challenges for pharmaceutical industry, researchers and formulation scientists is to improve dissolution and hence bioavailability of these drugs. ${ }^{2}$ Poor aqueous Solubility is creating troubles during formulation development, screening of new compounds and clinical testing for pharmacological activity and hence there is need of smart technology for formulations which will amplify aqueous solubility and thus bioavailability of drugs.

Drug nanocrystals are considered as a novel approach to improve the solubility of all poorly soluble drugs since the technique is simple and effective which can quickly launch product to the market. Nanocrystals provide distinctive features including enhancement of saturation solubility, dissolution velocity and adhesiveness to surface/cell membranes. Aggregation can be prevented by the addition of surfactant which can provide an electrostatic and steric repulsion between the nanocrystals. ${ }^{2,3}$ The main hitch of this approach is that drug nanocrystals are thermodynamically unstable and high surface energy lean to aggregate them during manufacturing, storage etc. ${ }^{4}$ Thus, development of stable nanocrystals of poorly soluble drug ensure its feasibility and is a pre-requisite. ${ }^{5}$ Nanocrystals can be prepared by precip-
Submission Date: 11-08-2018; Revision Date: 28-12-2018; Accepted Date: 16-03-2019

DOI: 10.5530/ijper.53.3.81 Correspondence: Dr. Namdeo R. Jadhav, Department of Pharmaceutics, Bharati Vidyapeeth College of Pharmacy, Kolhapur-416013, Maharashtra, INDIA.

Phone: +9102312637286 E-mail: nrjadhav18@rediffmail.com

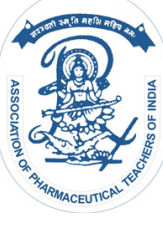

www.ijper.org 
itation, milling, high pressure homogenization and combination methods such as Nano-Edge ${ }^{\mathrm{TM}}$, Smart Crystal and Precipitation-lyophilization-homogenization (PLH) technology. ${ }^{6,7}$

Cocoons of silkworm Bombyx mori consist of proteins -Sericin and fibroin. ${ }^{8,9}$ Sericin amounts for $25-30 \%$ of cocoon. It is a globular protein soluble in water and has molecular weight ranging from 10 to $300 \mathrm{KDa} \cdot{ }^{10,11}$ and is composed structurally of 18 different amino acids most having strong polar groups such as carboxyl, hydroxyl and amino groups. High concentration of serine and aspartic acid gives sericin hydrophilic characters. ${ }^{8}$ Silk is produced in large amount all over the world, India and china being main producers. About 4,00,000 tons of cocoons are produced worldwide and from that 50,000 tons of sericin is removed by different processes and discarded as waste water. ${ }^{12-14}$ This causes environmental contamination due to its high oxygen demand for degradation. If waste sericin is recovered and recycled, it will not only reduce environmental pollution but can be used in different fields such as pharmaceutical, cosmetics, food industry, biomaterial manufacturing etc. ${ }^{15-}$ ${ }^{21}$ Due to high concentration of hydrophilic groups it shows excellent water retention property and these groups have strong polar side groups which imparts property of electrostatic bonding with drug molecules. ${ }^{22}$ This character is used to prepare and stabilize nanocrystals of poorly soluble drugs which shows enhanced dissolution rates and bioavailability. ${ }^{23}$ Strategies in preparation of these nanocrystals involves different methods such as milling, precipitation, high pressure homogenization etc. ${ }^{24}$ To exhibit its efficacy, nanocrystal of poorly soluble BCS-II drugs Hydrochlorothiazide were prepared by antisolvent precipitation techniques. ${ }^{25}$ Further this nanocrystals were evaluated by- Drug content, particle size, zeta potential, Infrared spectroscopy, X-ray diffraction, Differential scanning calorimetry, scanning electron microscopy, in vitro dissolution studies and stability study.

\section{MATERIALS AND METHODS}

\section{Materials}

Hydrochlorothiazide (HCTZ) was obtained as a gift sample from Abbott Laboratories, Goa, India. Sericin waste water was collected from Sericulture farm, Hupari, Maharashtra, India. All other chemicals were of analytical grade.

\section{Method}

\section{Recovery of Sericin}

Silk alkali degumming process generates sericin water which was collected and sericin was recovered from this sericulture waste water by the method mentioned with few modifications. This degummed Sericin water was concentrated at $60^{\circ} \mathrm{C}$ by heat vaporization so as to obtain aqueous sericin solution which was centrifuged at $5000 \mathrm{rpm}$ for $30 \mathrm{~min}$. and supernant was alienated. Obtained supernatant was filtered, purified against distilled water to removes remaining traces of slats if any and this purified Sericin solution by subjecting to lyophilization (Pressure $0.013 \mathrm{mbar}$ and temperature $-49^{\circ} \mathrm{C}$ ) was converted to sericin powder. This powder was then reduced to fine powder using mortar and pestle and was stored at $4^{\circ} \mathrm{C}$.

\section{Preparation of Hydrochlorothiazide Nanocrystals}

Hydrochlorothiazide nanocrystals were prepared by the anti-solvent precipitation method in following wayHydrochlorothiazide $60 \mathrm{mg}$ was dissolved in organic solvent acetone and $0.5 \%, 1 \%, 1.5 \%, 2 \%$ concentration of sericin was dissolved in water system (Antisolvent) for different batches. The anti-solvent was cooled to below $5^{\circ} \mathrm{C}$ in an ice-water bath. Then, drop wise addition of organic solution into $50 \mathrm{ml}$ of the pre-cooled anti-solvent at a stirring speed of $2000 \mathrm{rpm}$ was carried out for different batches. Nanocrystals were prepared by adding the micro liter quantity of drug solution to milliliter quantity of acetone quickly with ceaseless stirring on magnetic stirrer. Solvent was removed by stirring at for next 2 hrs. Then this nanocrystal liquid is lyophilized at pressure $0.013 \mathrm{mbar}$ and temperature $-49^{\circ} \mathrm{C} .^{7}$

\section{Percentage Yield of Lyophilized Process}

The amount of product obtained after completion of process is determined by percentage yield. Briefly powder obtained from lyophilized process was collected and product yield was obtained from following equation.

$$
\text { Percent yield }=\frac{\text { Practical yield }}{\text { Therotical yield }} \times 100
$$

\section{Particle Size Analysis}

Particle size of the nanocrystal's formulation was done by using particle size analyzer (Malvern Zetasizer Ver. 7.11 UK). Size and size distribution of the nanocrystals particles were determined through particle size analyzer with a wet sampling system and the diameters reported were calculated using mean particle size distribution.

\section{Zeta Potential (Ъ) Analysis}

The zeta potential of the nano suspended particles surface was determined by electrophoretic mobility in an apparatus such as a Malvern Zetasizer (Malvern Instruments, UK) equipped with suitable software and 
calibrated with the supplied standard. Three consecutive measurements are performed at $25^{\circ} \mathrm{C}$ using a constant cell drive of $150 \mathrm{mV}$. The electrophoretic mobility is converted into zeta potential values through the Smoluchowsky equation, using the dielectric constants and viscosity of dispersion medium.

\section{Percent Drug Content}

The lyophilize nanocrystal powder (10mg) was dissolved in $1 \mathrm{ml}$ methanol and volume was made up to $100 \mathrm{ml}$ in volumetric flask with methanol. $0.1 \mathrm{ml}$ of above solution was further diluted to $10 \mathrm{ml}$ methanol and analyzed by spectrophotometrically at $274 \mathrm{~nm}$. The Hydrochlorothiazide content in nanocrystals $(\% \mathrm{w} / \mathrm{w})$ was calculated using calibration curve.

\section{FTIR}

Infrared spectroscopy of pure drugs, sericin, physical mixtures and their nanocrystals were recorded using ATR-FTIR spectrophotometer (Bruker Alpha-T model in the region of $\left.4000-400 \mathrm{~cm}^{-1}\right)$.

\section{PXRD}

Powder X-ray diffraction pattern of the pure drugs, sericin and nanocrystals were obtained using Philips X-ray diffractometer, PW-3710, Holland, using $\mathrm{Cu} \mathrm{K} \alpha$ radiation $(\lambda=1.5405 \AA)$ at voltage of $40 \mathrm{kV}$ and $30 \mathrm{~mA}$ current). The data recorded over a range of $10^{\circ} \mathrm{C}$ to $50^{\circ} \mathrm{C}$ at a scanning rate of $5 \times 103 \mathrm{cps}$ using a chart speed of $5 \mathrm{~mm} / 2^{\circ} \mathrm{C}$.

\section{DSC}

Differential scanning calorimetry (DSC) studies were carried out using Mettle-Toledo DSC 821 instrument. Indium and zinc standards were used to calibrate the DSC temperature and enthalpy scale. The lyophilize nanocrystals of optimized batch and pure drug Hydrochlorothiazide are hermetically sealed in aluminum crucibles and heated at a constant rate of $10^{\circ} \mathrm{C} / \mathrm{min}$ over a temperature range of $25-300^{\circ} \mathrm{C}$. Inert atmosphere was maintained by purging nitrogen gas at flow rate of 50 $\mathrm{ml} / \mathrm{min}$.

\section{\% Crystallinity Study}

The percentages of crystalline material present in the samples were calculated using equation.

$\%$ Crystalinity $=\delta H m$ nanocrystal $\delta H m D r u g \mathrm{x} \mathrm{w} \mathrm{x}$ 100.

Where $\delta H m$ is the melting enthalpy $\left(\mathrm{J} \mathrm{g}^{-1}\right), \delta H m D r u g$ the melting enthalpy of $\operatorname{drug}\left(\mathrm{J} \mathrm{g}^{-1}\right)$ and $W$ is the weight fraction of drug in sericin.

\section{SEM}

Evaluation of particle size and morphology was achieved via scanning electron microscopy (SEM), using a JSM-6360 microscope (JEOL, Tokyo, Japan) at magnifications of $100 x$ and $1000 x$.

\section{In vitro Dissolution Study}

\section{Drug Dissolution from Nanocrystals}

The dissolution studies of hydrochlorothiazide and its all nanocrystals were performed using a US Pharmacopeia type I dissolution test apparatus (Electro lab).

The nanocrystals of all batches were filled into hard gelatin capsules. The HCTZ nanocrystal hard gelatin capsule placed into basket loaded with $900 \mathrm{ml}$ of 0.1 N HCL and phosphate buffer at $37 \pm 0.5^{\circ} \mathrm{C}$ with speed of $100 \mathrm{rpm}$. Each sample (5 ml) was withdrawn at 15, $30,45,60, \ldots ., 120$ min and 1, 2, 3, 4, 5, 6, 7, 8, 9, 10, 11, $12 \mathrm{hr}$ respectively with replacement by an equal volume of temperature-equilibrated media and filtered through $0.45 \mu \mathrm{m}$ pore size nylon filter. The amount of drug dissolved was determined by UV spectroscopy at $\lambda_{\max }$ of $274 \mathrm{~nm}$ for $0.1 \mathrm{~N}$ HCL and phosphate buffer $\mathrm{pH} 6.8$.

\section{Stability Studies}

Keep the filled capsule for stability study at two temperature conditions i.e $2-8^{\circ} \mathrm{C}$ and RT. The stability of Hydrochlorothiazide nanocrystals on optimized formulation P1were studied at $2-8^{\circ} \mathrm{C}$ and RT for 3 months according to ICH guidelines. Periodically, samples were withdrawn and the particle size as well as drug content was determined. They were withdrawn at specified intervals for analysis over a period of 30 days, 60 days and 90 day after which particle size and percent drug content were determined.

\section{RESULTS AND DISCUSSION}

\section{Recovery of Sericin}

Sericin was lucratively recovered from waste water by lyophilization and $22.5 \mathrm{~g}$ sericin powder was obtained. According to literature, silk cocoons contains about $20-30 \%$ sericin protein which indicates that sericin was entirely separated from silk fibroin in reeling process. Thus, yield of sericin powder was $75 \% \mathrm{w} / \mathrm{w}$ which was used for further course of action.

\section{Preparation of Hydrochlorothiazide Nanocrystals}

Hydrochlorothiazide nanocrystals were prepared by antisolvent precipitation method.

Four different batches of nanocrystals were prepared shown in Table 1. 


\begin{tabular}{|c|c|c|c|c|}
\hline \multirow[t]{2}{*}{ Ingredient } & \multicolumn{4}{|c|}{ Formulation } \\
\hline & P1 & P2 & P3 & P4 \\
\hline Hydrochlorothiazide (mg) & 60 & 60 & 60 & 60 \\
\hline Acetone (ml) & 50 & 50 & 50 & 50 \\
\hline Sericin (mg) & 30 & 60 & 90 & 120 \\
\hline Water (ml) & 50 & 50 & 50 & 50 \\
\hline
\end{tabular}

\begin{tabular}{|c|c|c|c|c|c|c|c|}
\multicolumn{6}{|c|}{ Table 2: \% Yield, Particle Size, PDI, Zeta Potential, \% Drug content, \% Crystallinity of all } \\
Formulation Batches. \\
\hline $\begin{array}{c}\text { Sr. } \\
\text { no }\end{array}$ & $\begin{array}{c}\text { Batch } \\
\text { Code }\end{array}$ & $\begin{array}{c}\text { Percentage } \\
\text { Yield }\end{array}$ & $\begin{array}{c}\text { Particle } \\
\text { Size (nm) }\end{array}$ & PDI & $\begin{array}{c}\text { Zeta } \\
\text { Potential } \\
\text { (mV) }\end{array}$ & $\begin{array}{c}\% \text { Drug } \\
\text { content }\end{array}$ & $\%$ crystallinity \\
\hline 1 & P1 & $\mathbf{7 5}$ & $\mathbf{2 5 1 . 8}$ & $\mathbf{0 . 7 3 7}$ & $\mathbf{- 1 8 . 8}$ & $\mathbf{9 3 . 6 6 \pm 0 . 5 1}$ & $\mathbf{5 . 2 6}$ \\
\hline 2 & P2 & 70 & 495.1 & 0.597 & -6.01 & $85.44 \pm 0.78$ & 14.25 \\
\hline 3 & P3 & 67 & 336.0 & 0.605 & -16.3 & $80.83 \pm 0.73$ & 3.49 \\
\hline 4 & P4 & 65 & 390.2 & 0.625 & -14.2 & $79.5 \pm 0.65$ & 7.67 \\
\hline
\end{tabular}

\section{Percentage Yield}

The percentage yield of Lyophilized process obtained with optimized parameter was in the range of $65-75 \%$. As P-1 batch was optimized as it showed high percentage yield. Yield of all Lyophilized formulation batches are as shown in Table 2.

\section{Particle Size Analysis and Zeta Potential}

Particle size and the Polydispersity index (PDI) of all the formulations were measured. It was found that the smallest particle size was observed in batch P1 as compared to other formulations. The zeta potential value of all the batches was found to be in between -6.01 to $-18.8 \mathrm{mV}$, optimized batch shows $-18.8 \mathrm{mV}$ mean zeta potential which means optimized batch have more stability than other batches as shown in Table 2 .

\section{Percent Drug Content}

The percent drug content of all prepared nanocrystals in various ratios of sericin was found to be between $79.5 \pm 0.65$ to $93.66 \pm 0.51 \%$. All the nanocrystals of different ratios showed the presence of high drug content which indicates that the drug is uniformly dispersed within the polymer matrix. Amongst all batches, P-1 showed higher percentage of drug content as shown in Table 2.

\section{FTIR}

The ATR-FTIR Spectra of each drug, sericin, physical mixtures and their optimized batch (P1) have been shown in Figure 1.
The characteristics peak of the Hydrochlorothiazide observed at $3260.11 \mathrm{~cm}^{-1}$ hydroxyl group (OH stretching), a band peak at $3356.54 \mathrm{~cm}^{-1}$ owing to imino group (N-H stretching), the characteristics peak of the carbonyl group $(\mathrm{C}=\mathrm{O}$ stretching) present in the amide group at $1594.66 \mathrm{~cm}^{-1}$, a band peak at 1462.55 $\mathrm{cm}^{-1}$ owing to $\mathrm{C}=\mathrm{C}$ stretching (Aromatic) confirm the presence of Hydrochlorothiazide.

The IR spectrum of physical mixture indicated principal peaks belonging to measure functional group such as $1064 \mathrm{~cm}^{-1}$ ( $\mathrm{S}=\mathrm{O}$ stretching), $1517 \mathrm{~cm}^{-1}(\mathrm{~N}-\mathrm{H}$ stretching), $1516.25 \mathrm{~cm}^{-1}$ ( $\mathrm{C}=\mathrm{C}$ aromatic stretching).

The optimized batch (P1) indicated principal peaks observed at $3260.94 \mathrm{~cm}^{-1}$ (OH stretching), a band peak at $3408.57 \mathrm{~cm}^{-1}\left(\mathrm{~N}-\mathrm{H}\right.$ stretching), $1524.88 \mathrm{~cm}^{-1} \mathrm{C}=\mathrm{C}$ stretching (Aromatic), $1655.01 \mathrm{~cm}^{-1}(\mathrm{C}=\mathrm{O}$ stretching), $1082.00 \mathrm{~cm}^{-1}(\mathrm{~S}=\mathrm{O}$ stretching $)$.

The intention behind compatibility studies of drug and polymer by FTIR is to study the chemical interaction between them, which may affect the results positively or negatively. An overlain spectrum of pure Hydrochlorothiazide and physical mixture is shown in Figure $7 .^{12}$ FTIR studies revealed that the fundamental peaks of Hydrochlorothiazide are retained in the physical mixture indicating absence of any chemical interaction between Hydrochlorothiazide and sericin. Thus, the sericin was used in the Hydrochlorothiazide nanocrystal formulation. From the FTIR studies, it can be seen that the principal peaks of the pure drug Hydrochlorothiazide retained in the optimized batches are almost identical. 


\section{PXRD}

PXRD spectra of Hydrochorothiazide show characteristic $2 \Theta$ peaks observed at $16^{\circ} \mathrm{C}, 19^{\circ} \mathrm{C}, 20^{\circ} \mathrm{C}, 24^{\circ} \mathrm{C}, 28^{\circ} \mathrm{C}$, $18^{\circ} \mathrm{C}$. Spectra showed sharp and intense diffraction peaks which are an indication that Hydrochorothiazide can be considered as crystalline in nature.

PXRD spectra of sericin, has showed diffraction peaks which are very low in intensity. Intensities of these peaks are very low so, it can be considered as less crystalline. The PXRD spectrums of a physical mixture of Hydrochlorothiazide with silk sericin are shown. The analysis of PXRD pattern reveals the intensity of the crystallinity of the pure drug and when it was combined with silk sericin. As physical mixture intensities of the peaks were same to the pure drug intensities, it inveterate that the Hydrochlorothiazide is compatible with silk sericin. PXRD of HCTZ and sericin-HCTZ nanocrystal optimized batch P1 were compared to detect the crystallinity and polymorphic transformation. The diffraction spectrum of pure HCTZ showed the drug was of crystalline nature as indicated by frequent relative sharp and distinct peaks at a diffraction angle of $2 \theta$. The nanocrystals of optimized batch P1 was characterized by same intensity of the diffraction peak, when compared to that of HCTZ. This clearly indicates insignificant reduction in the crystallinity of the precipitated HCTZ nanocrystals and the ordered crystals were majority and the crystalline state would contribute to the lower drug loading capacity. It was confirmed that HCTZ existed in crystalline state in the HCTZ nanocrystals because of the persistent sharp peak of HCTZ in the diffraction pattern as shown in Figure 2.

\section{DSC}

DSC thermogram of pure hydrochlorothiazide showed a sharp endothermic peak at $269.57^{\circ} \mathrm{C}$. From the thermogram it was obvious that hydrochlorothiazide is crystalline in nature.

The DSC thermo gram of silk sericin has showed an endothermic peak at $74.16^{\circ} \mathrm{C}$ and another near $232.5^{\circ} \mathrm{C}$

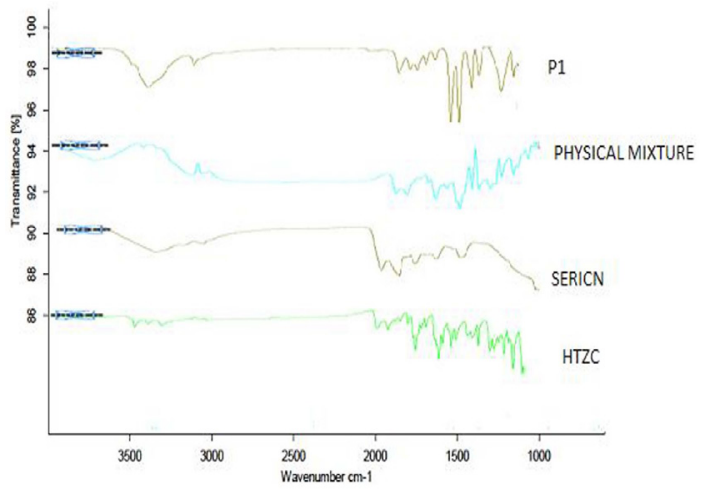

Figure 1: FTIR of Drug, Sericin, Physical Mixture of HCTZ and Optimized Batch P1.

\section{Table 4: In vitro Dissolution Data of Nanocrystals Formulations in Phosphate Buffer PH 6.8.}

\begin{tabular}{|c|c|c|c|c|c|}
\hline \multirow{2}{*}{$\begin{array}{l}\text { Sr. } \\
\text { No. }\end{array}$} & \multirow{2}{*}{$\begin{array}{c}\text { Time } \\
\text { (hr) }\end{array}$} & \multicolumn{4}{|c|}{$\%$ Drug Dissolution } \\
\hline & & P1 & P2 & P3 & P4 \\
\hline 1 & 0 & 0 & 0 & 0 & 0 \\
\hline 2 & 1 & $32.62 \pm 0.1$ & $30.88 \pm 0.5$ & $29.78 \pm 0.3$ & $31.59 \pm 0.2$ \\
\hline 3 & 2 & $37.39+0.4$ & $32.68 \pm 0.5$ & $31.65 \pm 0.3$ & $36.24 \pm 0.2$ \\
\hline 4 & 3 & $38.24 \pm 0.3$ & $36.75 \pm 0.4$ & $34.75 \pm 0.3$ & $37.56 \pm 0.4$ \\
\hline 5 & 4 & $40.13 \pm 0.6$ & $36.87 \pm 0.7$ & $38.84 \pm 0.2$ & $38.67 \pm 0.1$ \\
\hline 6 & 5 & $42.86 \pm 0.2$ & $45.87 \pm 0.1$ & $44.66 \pm 0.4$ & $45.83 \pm 0.3$ \\
\hline 7 & 6 & $51.75 \pm 0.1$ & $46.66 \pm 0.1$ & $44.78 \pm 0.1$ & $45.96 \pm 0.7$ \\
\hline 8 & 7 & $52.29 \pm 0.6$ & $48.92 \pm 0.1$ & $52.82 \pm 0.2$ & $48.78 \pm 0.1$ \\
\hline 9 & 8 & $55.54 \pm 0.5$ & $53.01 \pm 0.5$ & $59.02 \pm 0.4$ & $49.76 \pm 0.5$ \\
\hline 10 & 9 & $56.88 \pm 0.5$ & $55.15 \pm 0.1$ & $62.45 \pm 0.5$ & $52.5 \pm 0.4$ \\
\hline 11 & 10 & $59.63 \pm 0.2$ & $71.21 \pm 0.3$ & $70.22 \pm 0.5$ & $53.95 \pm 0.8$ \\
\hline 12 & 11 & $79.46 \pm 0.1$ & $76.92 \pm 0.2$ & $75.22 \pm 0.1$ & $78.61 \pm 0.6$ \\
\hline 13 & 12 & $89.39+0.4$ & $80.28 \pm 0.2$ & $82.88 \pm 0.1$ & $85.59 \pm 0.1$ \\
\hline
\end{tabular}

Table 3: In vitro Dissolution Data of Nanocrystals Formulation in 0.1 N HCL.

\begin{tabular}{|c|c|c|c|c|c|c|}
\hline \multirow{2}{*}{ Sr. No. } & $\begin{array}{c}\text { Time } \\
(\mathbf{m i n})\end{array}$ & \multicolumn{5}{|c|}{ \% Drug dissolved } \\
\cline { 3 - 7 } & & Standard (drug) & $\begin{array}{c}\mathbf{P 1} \\
(\mathbf{1 : 0 . 5 )}\end{array}$ & $\begin{array}{c}\mathbf{P 2} \\
\mathbf{( 1 :} \mathbf{1})\end{array}$ & $\begin{array}{c}\mathbf{P 3} \\
\mathbf{( 1 : 1 . 5 )}\end{array}$ & $\begin{array}{c}\mathbf{P 4} \\
\mathbf{( 1 : 2 )}\end{array}$ \\
\hline 1 & 0 & 0 & 0 & 0 & 0 & 0 \\
\hline 2 & 15 & 34.44 & $3.17 \pm 0.2$ & $3.29 \pm 0.4$ & $2.03 \pm 0.8$ & $2.25 \pm 0.4$ \\
\hline 3 & 30 & 47.12 & $5.15 \pm 0.4$ & $4.30 \pm 0.2$ & $3.97 \pm 0.5$ & $3.25 \pm 0.2$ \\
\hline 4 & 60 & 78.23 & $7.12 \pm 0.5$ & $6.98 \pm 0.2$ & $6.78 \pm 0.5$ & $7.46 \pm 0.1$ \\
\hline 5 & 90 & 83.01 & $11.5 \pm 0.4$ & $9.66 \pm 0.1$ & $9.66 \pm 0.1$ & $10.6 \pm 0.4$ \\
\hline 6 & 120 & 92.60 & $14.6 \pm 0.2$ & $12.3 \pm 0.3$ & $12.3 \pm 0.1$ & $13.7 \pm 0.4$ \\
\hline
\end{tabular}




\begin{tabular}{|c|c|c|c|c|}
\hline \multicolumn{5}{|c|}{ Table 5: Stability of HCTZ Loaded Nanocrystals (Optimized Batch P1) } \\
at RT and 2-8 ${ }^{\circ} \mathbf{C}$. \\
\hline Time & Initial & 1 month & 2 months & 3 months \\
\hline $\begin{array}{c}\text { Drug content } \\
\left.\text { (\%) (2-8 }{ }^{\circ} \mathbf{C}\right)\end{array}$ & $93.66 \pm 0.21$ & $93.01 \pm 0.45$ & $93.44 \pm 0.01$ & $93.03 \pm 0.35$ \\
\hline $\begin{array}{c}\text { Drug content } \\
\text { (\%) (RT) }\end{array}$ & $94.01 \pm 0.22$ & $94.5 \pm 0.48$ & $93.4 \pm 0.55$ & $93.55 \pm 0.26$ \\
\hline $\begin{array}{c}\text { Particle size } \\
\left.\text { (nm) (2-8 }{ }^{\circ} \mathbf{C}\right)\end{array}$ & $251.14 \pm 11.5$ & $258.67 \pm 12.5$ & $260.16 \pm 16.3$ & $262.44 \pm 14.0$ \\
\hline $\begin{array}{c}\text { Particle size } \\
\text { (nm) (RT) }\end{array}$ & $261.8 \pm 7.3$ & $278.3 \pm 12.5$ & $285.6 \pm 11.2$ & $295.01 \pm 8.12$ \\
\hline $\begin{array}{c}\text { Zeta potential } \\
\left(\mathbf{2}-8^{\circ} \mathbf{C}\right)\end{array}$ & -18.8 & -18.8 & -18.8 & -18.8 \\
\hline $\begin{array}{c}\% \text { Crystallinity } \\
\text { (RT) }\end{array}$ & 44.01 & 44.15 & 45.12 & 45.07 \\
\hline
\end{tabular}

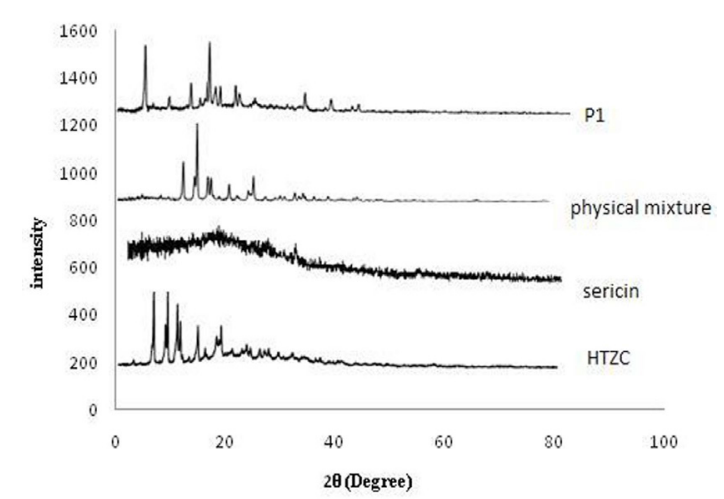

Figure 2: PXRD of Drug, Sericin, Physical Mixture of HCTZ and Optimized Batch P1.

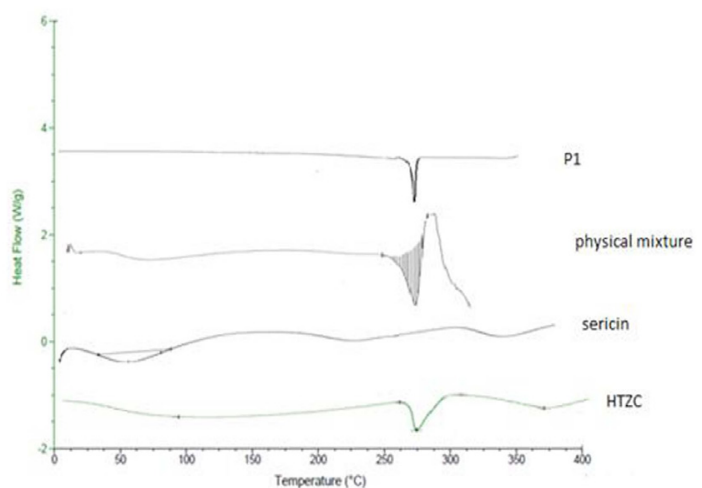

Figure 3: DSC of Drug, Sericin, Physical Mixture of HCTZ and Optimized Batch P1.

were observed. The former peak signifies the molecular mobility and melting which is induced thermally, while the latter indicates thermal decomposition.

The thermal stability of sericin is influenced by the use of chemicals during the extraction process. Bombyx mori indicates low thermal stability due to low degradation temperature.

DSC of physical mixture shows that there were no substantial fluctuations in the peak position in Hydrochlorothiazide melting endotherm. HCTZ and Sericin physical mixture shows sharp endothermic peak at $269^{\circ} \mathrm{C}$. From this it was concluded that there was no interaction found between HCTZ and Sericin and they are compatible with each other.

The DSC results of the Hydrochlorothiazide pure drug and the formulation P1. The pure drug exhibited a large and sharp endothermic peak at $269.57^{\circ} \mathrm{C}$ indicating the melting point. DSC thermo gram of formulation P1 showed an endothermic peak at $270^{\circ} \mathrm{C}$ ascribed to the melting of Hydrochlorothiazide indicated the slight change in the crystalline nature. However, no additional peaks were found to demonstrate the significant changes in the melting characteristics of Hydrochlorothiazide in the formulation indicating no polymorphic changes in the Hydrochlorothiazide. The peaks were found to be nearly identical as sown in Figure 3.

\section{$\%$ Crystallinity Study}

From the melting enthalpy of pure drug and its respective antisolvent nanocrystals, crystallinity of sample can be determined. The percent crystallinity of all prepared nanocrystals in various ratios of sericin was found to be between 26.40 to $44.01 \%$.

\section{SEM}

The SEM images and morphology of the Hydrochlorothiazide nanocrystals (P1) are presented. The nanocrystals were found to be sharp needle shaped with a narrow particle size distribution as shown in Figure 4. 


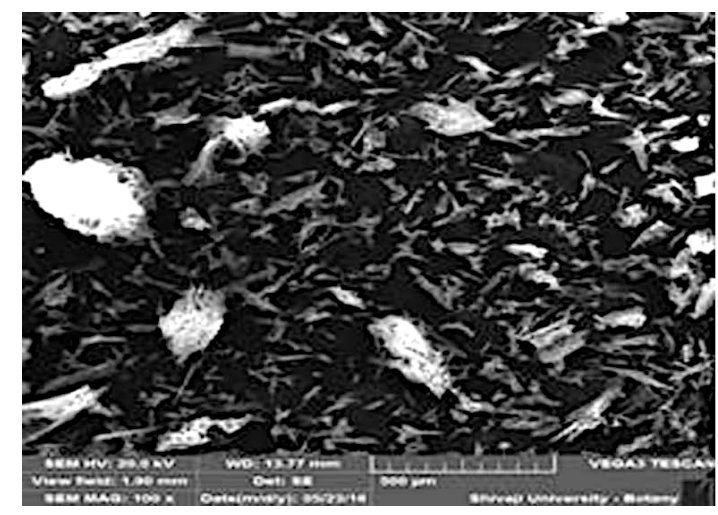

Figure 4: SEM Images of Optimized Batch P1 at 100.

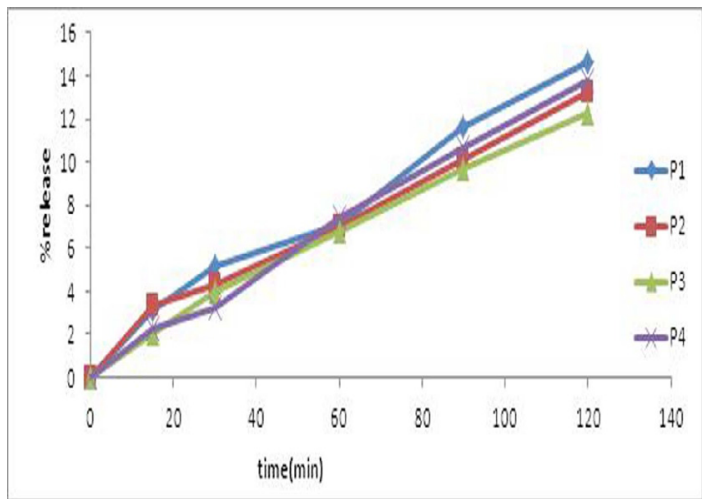

Figure 5: Plot of \% drug Dissolution of different batches of Nanocrystal Formulations in $0.1 \mathrm{~N} \mathrm{HCL}$.

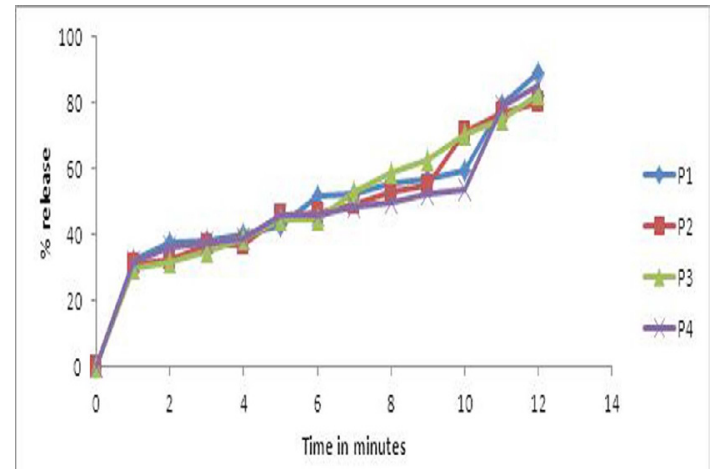

Figure 6: Plot of \% Drug Dissolution of Different Batches of Nanocrystal Formulations in Phosphate Buffer PH 6.8.

\section{In vitro Dissolution Study}

The in vitro dissolution profile of all nanocrystal formulations in dissolution media $0.1 \mathrm{~N} \mathrm{HCl}$ and phosphate Buffer pH 6.8 is shown in Table 3 and Table 4. Nanocrystal formulation batch P1 showed significantly higher $\%$ dissolution of drug as compared with that of all remaining batches. The dissolution profile of all batches in $0.1 \mathrm{~N}$ HCL is shown in Figure 5. The percentage dissolution of optimized batch P1 was observed $89.39 \%$ as shown in Figure 6.

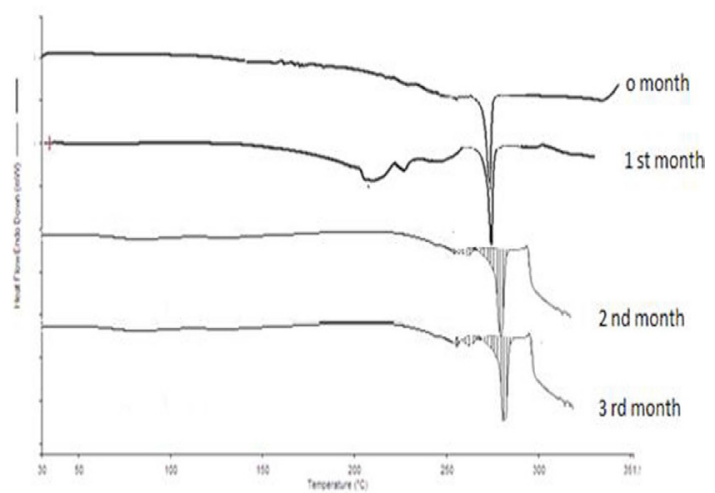

Figure 7: Stability DSC of Initial to Three Month.

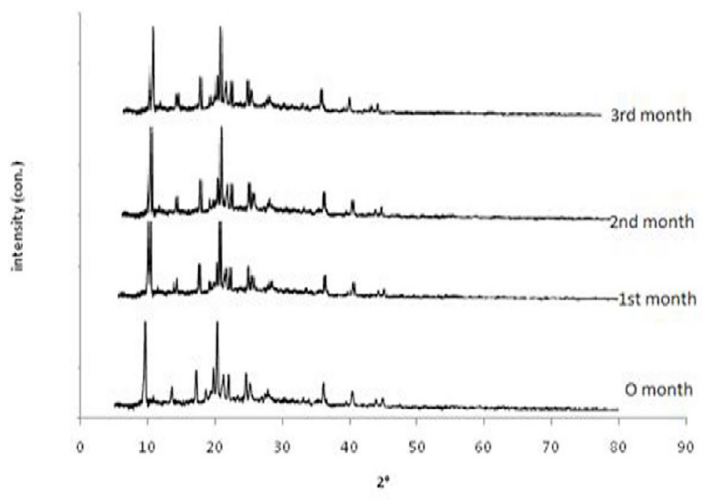

Figure 8: Stability PXRD of Initial to Three Month.

From dissolution profile it was observed that when capsule containing HCTZ silk sericin nanocrystal (Batch P1) was placed in dissolution medium it first get swells followed by drug dissolution over an extended period of time from the peppas formed by silk sericin. It can be concluded that silk sericin imparts good peppas integrity as for extended drug dissolution. From the dissolution studies of the formulations, formulation of batch HCTZ-sericin nanocrystal filled capsule showed better drug dissolution with extended dissolution and good peppas integrity throughout dissolution period. Hence, formulation P1 is considered as the best formulation.

\section{Stability Studies}

The stability of Hydrochlorothiazide loaded nanocrystals in terms of drug content, particle size distribution, zeta potential, $\%$ crystallinity was monitored for 3 months at $2-8^{\circ} \mathrm{C}$ and RT $\left(25-30^{\circ} \mathrm{C}\right)$. The nanocrystals showed physical stability for a period of 3 months at refrigerated conditions. The particle size, drug content, zeta potential, $\%$ crystallinity of the nanocrystals at different time interval. At room temperature, Particle size was increased and drug content was found to be decreased. It was found that no noteworthy difference was observed in the particle size and drug content of 
nanocrystals after 3 months at refrigerated conditions indicated its suitability for storage at $2-8^{\circ} \mathrm{C}$.

Zeta potential remains constant at $2-8^{\circ} \mathrm{C}$ indicating no significant differences. \% crystallinity of nanocrystals was slightly increased but not any noteworthy difference after 3 months storage as shown in Table 5.

\section{CONCLUSION}

From this study we conclude that Hydrochlorothiazide has been effectively converted to nanocrystals. The P1 batch was optimized by performing particle size, zeta potential, XRD, DSC and results obtained shown that the nanocrystals are effectively stabilized. The Drug release from optimized batch P1was found to be $89.39 \%$. Superior release was observed when HCTZ nanocrystals were prepared by using sericin. Stability study of optimized batch has been given excellent stability results as shown in Figure 7 and 8. Percent Crystallinity of nanocrystals was somewhat increased but not any noteworthy change (44.01 to 45.07). As sericin is available at cheaper cost as an excipient, it has potential to revolutionise economics of sericulture as well as pharmaceutical industry. Thus, the sericin holds a great future for its use as stabilizer of nanocrystals in the formulations.

\section{ACKNOWLEDGEMENT}

Authors would like to thank to Dr. H. N. More, Principal of Bharati Vidyapeeth College of Pharmacy, Kolhapur for providing facilities to carry out our research work.

\section{ABBREVIATIONS}

UV: Ultra-Violet Spectroscopy; FTIR: Fourier Transform Infrared Spectroscopy; DSC: Differential Scanning Calorimetry; XRD: X-ray Diffractometry; PI: Polydispersity Index; SEM: Scanning Electron Microscopy; BCS: Biopharmaceutical Classification System; HCTZ: Hydrochlortiazide.

\section{REFERENCES}

1. Lipinski CA. Poor aqueous solubility- an industry wide problem in drug discovery. Am Pharm. 2002;5(3):82-5.

2. Patel AP, Patel JK, Deshmukh AB, Mishra BR. A Review on drug Nanocrystal a Carrier Free Drug Delivery. IJRAP. 2011;2(2):448-58.

3. Rajput $S$, Singh M. Sericin-A Unique Biomaterial. IOSR Journal of Polymer and Textile Engineering. 2015;2(3):29-35.
4. Peltonen L, Strachan C. Understanding Critical Quality Attributes for Nanocrystals from Preparation to Delivery. Molecules. 2015;20(12):22286300 .

5. Izquierdo P, Esquena J, Tadros TF, Dederen C, Garcia MJ, Azemar N, et al. Formation and stability of nano-emulsions prepared using the phase inversion temperature method. Langmuir. 2001;18(1):26-30.

6. Katteboinaa S. Drug Nanocrystals: A novel formulation approaches for poorly soluble drug. International Journal of Pharmatech Research. 2009;1(3):68294.

7. Aramwit $\mathrm{P}$, Siritientong $\mathrm{T}$, Srichana $\mathrm{T}$. Potential applications of silk sericin, a natural protein from textile industry by-products. Waste Manag Res. 2012;30(3):217-24.

8. Takasu Y, Yamada H, Tsubouchi K. Isolation of three main sericin components from the cocoon of the silkworm, Bombyx mori. Biosci Biotechnology Biochem. 2002;66(12):2715-8.

9. Zhang Y, Ma Y, Xia Y, Shen W, Mao J. Silk Sericin insulin bioconjugates: synthesis, characterisation and biological activity. Journal of Controlled Release. 2006;115(3):307-15.

10. Zhang Y. Applications of natural silk protein sericin in biomaterials. Biotechnology Adv. 2002;20(2):91-100.

11. Tao W, Li M, Xie R. Preparation and structure of porous silk sericin materials. Macromol Mater Eng. 2005;290(3):188-94.

12. Aramwit P. Effectiveness of Inflammatory Cytokines Induced by Sericin Compared to Sericin in Combination with Silver Sulfadiazine Cream on Wound Healing. Wounds. 2009;21(8):198-206.

13. Rajput SK, Singh M. Sericin - A unique biomaterial. IOSR J Polym Text Eng. 2015;22:181-348.

14. Varaporn BJ, Boontida M. Nanocrystals for enhancement of oral bioavailability of poorly water-soluble drugs. Asian Journal of Pharmaceutical Sciences. 2015;10(1):13-23.

15. Zhaorigetu S, Yanaka N, Sasaki M, Watanabe H, Kato N. Inhibitory effects of silk protein- sericin on UVB-induced acute damage and tumor promotion by reducing oxidative stress in the skin of hairless mouse. $J$ Photochem Photobiol B Biol. 2003;71(1-3):11-7.

16. Tsubouchi K, Igarashi $Y$, Takasu $Y$, Yamada H. Sericin enhances attachment of cultured human skin fibroblasts. Biosci Biotechnol Biochem. 2005;69(2):4035.

17. Aramwit $\mathrm{P}$, Keongamaroon $\mathrm{O}$, Siritientong $\mathrm{T}$, Bang $\mathrm{N}$, Supasyndh $\mathrm{O}$. Sericin cream reduces pruritus in hemodialysis patients: a randomized, double-blind, placebo controlled experimental study. BMC Nephrol. 2012;13(1):13-119.

18. Kitisin T, Maneekan P, Luplertlop N. In-vitro Characterization of Silk Sericin as an Anti- aging Agent. J Agric Sci. 2013;5(3):54-63.

19. Wei HY, Jianping $Q$, Lingyun $C$, Fuqiang $H$, Wei W. Food proteins as novel Nanosuspension stabilizers for poorly water-soluble drugs. International Journal of Pharmaceutics. 2013;441(1-2):269-78.

20. Lee JH, Song DW, Park YH, Um IC. Effect of residual sericin on the structural characteristics and properties of regenerated silk films. Int J Biol Macromol. 2016;89:273-8.

21. Watthanaphanit A, Rujiravanit R. Sericin-binded-deprotenized natural rubber film containing chitin whiskers as elasto-gel dressing. Int J Biol Macromol. 2017;101:417-26.

22. Acarturk F, Kislal $\mathrm{O}$, Celebi $\mathrm{N}$. The effect of some natural polymers on the solubility and dissolution characteristics of nifedipine. Int $\mathrm{J}$ Pharm. 1992;851(1-3):1-6.

23. Padamwar MN, Pawar AP. Silk sericin and its applications: A review. Journal of Scientific and Industrial Research. 2004;63:323-9.

24. Pande VV, Abhale V. Nanocrystal Technology: A Particle Engineering Formulation Strategy for the poorly water-soluble drugs. Scholors Research Library Der Pharmacia Lettre. 2016;8(5):384-92.

25. Wu JH, Wang Z, Xu SY. Preparation and characterization of sericin powder extracted from silk industry wastewater. Food Chem. 2007;103(4):1255-62.

Cite this article: Giri $\mathrm{P}$, Jadhav $\mathrm{P}$, Patil U, Jadhav N. Hydrochlorothiazide Nanocrystals Stabilization by Silk Sericin. Indian J of Pharmaceutical Education and Research. 2019;53(3):493-502. 


\section{PICTORIAL ABSTRACT}

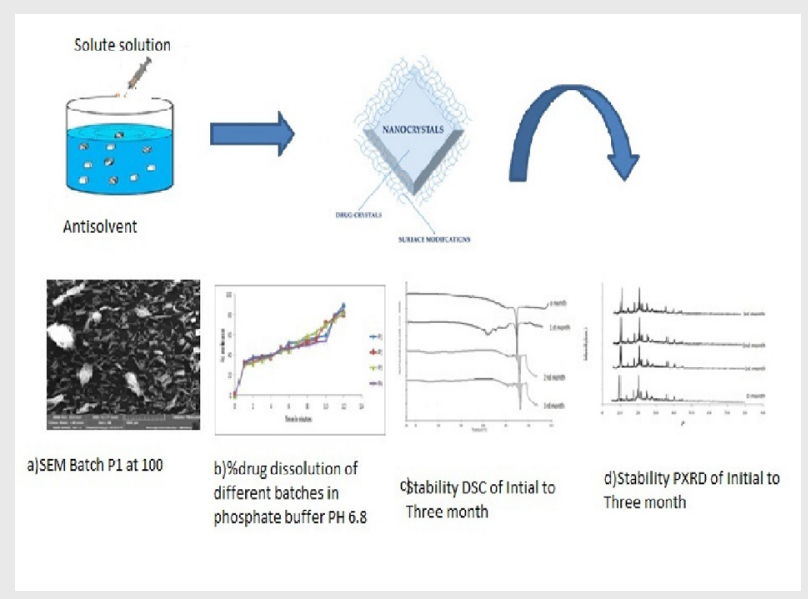

About Author

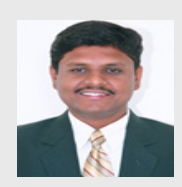

Dr. N. R. Jadhav is presently working as a Professor and Head, Dept. of Pharmaceutics at Bharati Vidyapeeth College of Pharmacy, Kolhapur. He is recipient of Barr. P. G. PATIL Ideal Teacher Award 2013, Shivaji University, Kolhapur. He has total teaching and research experience of 21 years. He has published many research articles in national and international journals and also worked as reviewer to reputed journals. He is a member of Board of Studies and Member of Research Advisory committee of Shivaji University, Kolhapur.

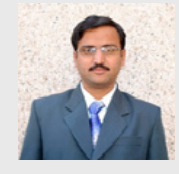

Mr. Udaykumar Patil has M. Pharm in Biopharmaceutics and pursuing Ph.D. in Pharmaceutical Sciences from Shivaji University, Kolhapur. He has more than 10 years of teaching experience and 6 years research experience. He is currently working as Assistant Professor, Dept. of Pharmaceutics at Bharati Vidyapeeth College of Pharmacy, Kolhapur, Maharashtra, India.

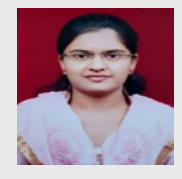

Ms Priyanka Giri has completed M. Pharmacy in Pharmaceutics from Bharati Vidyapeeth College of Pharmacy, Kolhapur, Maharashtra, India.

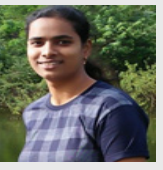

Ms Poonam Jadhav is pursuing degree of B. Pharmacy from Bharati Vidyapeeth College of Pharmacy, Kolhapur, Maharashtra, India and currently she is studying in $3^{\text {rd }}$ year B. Pharmacy.

\section{SUMMARY}

- The poor aqueous solubility of drugs (BCS Class II) is a major problem which limits the development of highly potent pharmaceuticals. Drug nanocrystals are considered as a novel approach to improve the solubility of all poorly soluble drugs. The main aim of the present study was screening of sericin for stabilization of drug nanocrystals.

- Silk sericin was used as nanocarrier based on its properties like biocompatibility, biodegradability, non- immunogenic, soluble in alkaline $\mathrm{pH}$ etc. Silk sericin was extracted from cocoons of B. mori by alkali salt method and purified by centrifugation, filtration and dialysis. The isoelectric $\mathrm{pH}$ of sericin was found to be at 3.9. Spectroscopic characterization of sericin by ATR-FTIR indicated presence of amide I ( $\mathrm{C}=\mathrm{O}$ stretching), amide II (N-H bending), amide III (C-N stretching) and amide IV. PXRD spectra of sericin showed its amorphous nature.

- Silk sericin nanocrystals containing hydrochlorothiazide were prepared by antisolvent precipitation technique. Prepared nanocrystals were lyophilized. Such nanocrystals were stable with small particle size and maximum percentage of drug content. Effect of protein concentration i. e. silk sericin concentration and stirring time on particle size, zeta potential, drug content was studied. Based on the best results obtained from batch P1 to P4, batch P1 was selected as an optimized batch for further study. Nanoparticles showed particle size of $251.8 \mathrm{~nm}$ with narrow size of distribution, $93.66 \pm 0.51$ drug content and $-18.8 \mathrm{mV}$ zeta potential. From the SEM images of prepared Hydrochlorothiazide loaded silk sericin nanocrystals it was observed that particles were needle in shape. ATR-FTIR analysis showed no drug polymer interactions. DSC and XRD studies revealed entrapment of Hydrochlorothiazide in silk sericin nanocrystals. Stability study of prepared nanocrystal showed no significant change in $\%$ Crystallinity and drug content. Thus, it was concluded that sericin enhances the solubility and dissolution of all poorly soluble drugs. Its role as a cheap and cost effective pharmaceutical excipient can be underlined, which holds potential to revolutionize economics of sericulture as well as pharmaceutical industry. Thus, sericin holds a great future for its use as stabilizer of nanocrystal in the formulations. 